\title{
Behaviour of geotextiles designed for pervious pavements as a support for biofilm development
}

Joseba R. Bayon, Daniel Jato-Espino, Elena Blanco-Fernandez, Daniel Castro-

Fresno*

GITECO Research Group, Civil Engineering School, Universidad de Cantabria, 39005 Santander, Spain

E-mail addresses: Joseba Rodriguez@donostia.org (Joseba R. Bayon), jatod@unican.es (D. Jato-

Espino), blancoe@unican.es (E. Blanco-Fernandez), castrod@unican.es (D. Castro-Fresno).

* Corresponding author. Tel.: +34 942202053; fax: +34 942201703.

\section{ABSTRACT}

Runoff is one of the main sources of contamination in urban areas, since water can transport pollutants from many different media, among which the hydrocarbons generated by vehicles have an especially significant relevance. Under this premise, the study of geotextiles used as water purification layers within pervious pavement structures becomes a crucial issue, since these fabrics have proved to be a suitable environment for the development of biofilms, which are groups of microorganisms capable of reducing the presence of hydrocarbons. For this reason, this paper proposes a first test to assess the capacity of ten different geotextiles by immersing them in a culture medium prepared to favour the growth of a microbial community. The results showed the major importance of geotextile thickness in the generation of biomass and its relationship to the manufacturing process of the fabric, either heatsealing or needle-punching. Consequently, a second test was developed to measure the mechanical behaviour of a geotextile of each type when buried in a microbially active soil under different conditions. The comparison between exposed and control samples revealed the maintenance of strength-related properties of geotextiles in the presence of microorganisms. 


\section{Keywords}

Geosynthetics; Geotextiles; Biofilm; Microorganisms; Pervious pavements; Hydrocarbons

\section{Introduction}

Pervious pavements constitute a widely applied solution to manage runoff in urban spaces, with the aim of both facilitating the infiltration of water and improving its quality in purification terms. These structures usually consist of a series of layers, among which geotextiles play an essential role in water treatment, especially with regard to pollutant removal (Castro-Fresno et al. 2013; Nnadi et al. 2014). Hydrocarbons are, in turn, one of the main sources of pollution in urban spaces, since their emissions result from incomplete combustion in vehicle engines (U.S. EPA 1994). Although there is experience in incorporating microorganisms in pavements to facilitate the degradation of hydrocarbons, later studies showed that such inoculation was not necessary (Pratt et al. 1999; Newman et al. 2002). Indeed, both oil and the materials forming the pervious pavement structure fulfil the required conditions to naturally support the growth of biological activity. Moreover, additional contributions from the air and other natural sources can also favour the establishment of an adequate microbial community.

Nevertheless, some authors (Bond et al. 1999) experimented with fertilizers inoculation, either granular or liquid, in order to accelerate the process of degradation and better control oil removal rates. Thus, better yields in hydrocarbon biodegradation were achieved with the use of slow release granular fertilizers, which proved able to remove oil in a retention period of seven months, almost a third of the time required by a liquid fertilizer. Later, the presence of biofilm was visually confirmed through microscopic examination of the microbial community developed in geotextiles when inoculated with microorganisms (Coupe et al. 2003). Thereby, despite the natural 
disposition of the system to favour the development of biofilm, these results suggest that the process can be optimized if a fertilizer is added to geotextiles.

Given the importance of the biofilm effect on urban runoff purification, this paper is focused on studying its interaction with geotextiles through two different laboratory tests. In the first, the geotextiles capacity to support biofilm growth is discussed by analysing how the development of the latter is affected by the specifics and physical features of these fabrics when submerged in a broth designed to stimulate the generation of biomass. The second experiment assesses the influence of microbiological activity on the geotextiles mechanical response by calculating variations in tensile strength and elongation after being exposed to the presence of microorganisms. Therefore, the aim of the paper is twofold: firstly, to identify geotextilerelated factors that most favour biofilm growth and development; secondly, to analyse the mechanical behaviour of these fabrics in the presence of a microbial community.

\section{Experimental methodology}

\subsection{Test 1. Biofilm growth and development on geotextiles}

A representative group of available and commonly used geotextiles in road construction in Spain was subject to a liquid bath of known characteristics in a plastic tank, with the aim of determining which provide better support to the biofilm growth. Although this experiment did not reflect the real situation of a pervious pavement structure, it was expected to provide reliable information on the response of geotextiles to a microbial environment.

Based on previous studies of the preparation of liquid media to cultivate biofilms for degradation purposes (Aleksieva et al. 2002; Li et al. 2005), the solution used was developed as shown in Figure 1 (I.C.T 2003).

Figure 1. Preparation of the liquid medium in which the biofilm was grown on the tested geotextiles 
This liquid was introduced into a plastic tank with dimensions $892 \mathrm{~mm} \times 302 \mathrm{~mm}$ and a height of $178 \mathrm{~mm}$ (see Figure 2). Ten different geotextiles of $65 \times 39 \mathrm{~mm}$ and characteristics as listed in Table 1 were placed in six parallel lines, such that three replicas of each of them hung from each support, resulting in thirty pieces of geotextile for every two lines. Aerobic conditions for the whole system were maintained using a perforated plastic pipe through which air was blown using a pump. Translucent areas of the tank were covered to prevent any entry of light susceptible of altering the solution. The liquid medium was circulated from one end of the tank to another, in order to ensure homogeneous mixing of the liquid.

Figure 2. Front and plan view of the experimental set up

Table 1. Geotextiles characteristics

Entire lines of samples were extracted at different times during the experiment $(15,30$ and 52 days) to determine the biofilm mass formed on the ten geotextiles by weight difference between the samples before and after testing using a scale.

\subsection{Test 2. Geotextiles resistance to microbiological degradation}

As a continuation of Test 1, this experiment was designed to study the influence of biofilm growth and presence of microorganisms on the mechanical properties of geotextiles. For this purpose, a test based on the standards UNE-EN 12225:2001 (AENOR 2001a), UNE-EN 12226:2001 (AENOR 2001b) and UNE-EN ISO 117211:2001 (AENOR 2001c) was developed. The test was based on the former, the two latter standards being used as a cross reference. The aim of standard UNE-EN 12225:2001 is to determine the microbiological resistance of geotextiles by a burial test in which at least 5 test specimens are exposed for 16 weeks to a microbially active soil under specified conditions (AENOR 2001c). At the end of the exposure, the test specimens are tested by comparing their physical properties with those obtained on at least 5 other unexposed specimens. The results are expressed as the percentage of retained strength or elongation, according to (AENOR 2001b). 
Under these premises, the proposed test was developed as described below.

\subsubsection{Materials}

The geotextiles to perform the test were selected for their representativeness, according to two different criteria: (1) their manufacturing process and (2) how widespread they were in use. Thus, Polyfelt TS-30 (needle-punched) and Inbitex (heatsealed) were chosen. They are the most widely used geotextiles in Spain and the United Kingdom, respectively, to the knowledge of the authors where geotextiles are used within permeable pavement structures. The physical characteristics of Inbitex are given in Table 1, as they are equivalent to those of the commercially available geotextile in Spain as Danofelt PP 125.

The soil used to bury these geotextiles was a 70:30 mixture of substrate taken from the field (topsoil and compost) and horse manure acting as a fertilizer, in order to meet the recommendations of standard UNE-EN ISO 11721-1:2001 (AENOR 2001c). To check the biological activity of the mixture, a series of cotton strips $100 \times 25 \mathrm{~mm}$ were buried in the soil for a period of 7 days. According to standard UNE-EN 12225:2001 (AENOR 2001a), the soil must be replaced by a mixture more active biologically if the tensile strength of the cotton strips after 7 days is over $\leq 25 \%$ of their original tensile strength. To carry out the geotextile test, this mixture was placed in containers of size and shape such that they allowed the burial of geotextile samples of $300 \mathrm{~mm}$ length and $50 \mathrm{~mm}$ width to a depth of between $100 \mathrm{~mm}$ and $150 \mathrm{~mm}$ (AENOR 2001a).

\subsubsection{Treatments}

The main difference between the proposed tests and the standard UNE-EN 12225:2001 was in standardising burial conditions to the specific tests to be conducted. Since the objective was to simulate the impact of microorganisms on geotextiles in the presence of hydrocarbons, the burial conditions of the samples were adapted by tripling the treatments as follows: 
(1) Geotextile samples were simply buried in the soil mixture, as stated in standard UNE-EN 12225:2001.

(2) Before being buried, the samples were impregnated with a mix of oil and distilled water at a concentration of $450 \mathrm{mg} / \mathrm{l}$, which is 100 times higher than that present in urban runoff, according to previous literature (Pratt et al. 1999). Such a concentration represented the accidental release or accumulation of a significant amount of oil in the medium, which may lead to clogging of the geotextile.

(3) Instead of applying the oil-water mix to the samples, it was distributed as homogenously as possible throughout the soil in the same concentration. Then, the samples were buried in the resulting mixture.

\subsubsection{Storage}

Three replicas of each geotextile were placed in a container along with a cotton strip to detect any decrease in biological activity during the test, in which case nutrients were added to reactivate the process. Thus, twelve containers (six for each geotextile) were stored in an incubator, divided into the three types of treatment described above.

Acting as controls, twenty-four geotextile samples were not buried, in order to compare their mechanical properties with those of the buried samples. Half of them were stored in a chamber under a controlled temperature of $22 \pm 2^{\circ} \mathrm{C}$ and relative humidity of $65 \pm$ $5 \%$, as specified in standard UNE-EN 12226:2001. The remaining samples were stored in a fridge at $4^{\circ} \mathrm{C}$ in a dry atmosphere, thus inhibiting biological activity. Half of the control strips were impregnated with oil and the other half were not.

Therefore, a total of forty-two samples of each geotextile were used, eighteen buried in the containers and twenty-four outside, twelve refrigerated and the other twelve under the atmospheric conditions specified by standard UNE-EN 12226:2001.

\subsubsection{Evaluation tests}


After sixteen weeks in an incubator checking the soil conditions every month, the buried samples were extracted from the containers and remaining particles of the soil mixture removed. Then, both exposed and control samples were immersed in a 70:30 mixture of ethanol and water for 300 seconds. Finally, the samples were cleaned with running water, rinsed with absorbent paper and dried for at least 72 hours at $20 \pm 2{ }^{\circ} \mathrm{C}$ and $65 \pm 5 \%$ relative humidity.

To estimate the variations of tensile strength and elongation, residual values were calculated using the following equations, pursuant to standard UNE-EN 12226:2001:

$$
\begin{aligned}
& \mathrm{R}_{\mathrm{f}}=\frac{\mathrm{F}_{\mathrm{e}}}{\mathrm{F}_{\mathrm{c}}} \times 100 \\
& \mathrm{R}_{\varepsilon}=\frac{\varepsilon_{\mathrm{e}}}{\varepsilon_{\mathrm{c}}} \times 100
\end{aligned}
$$

Where $R_{f}$ and $R_{\varepsilon}$ are \% residual strength and residual elongation, whilst $F$ and $\varepsilon$ are tensile strength and elongation at maximum load and the subscripts "e" and "c" refer to exposed and control samples, respectively.

\subsection{Statistical methods}

To interpret the results of both laboratory tests, a methodology based on both inferential and descriptive statistics was followed:

\subsubsection{Inferential statistics}

Inferential statistics are concerned with making predictions about the behaviour of an entire population from observations of a subset of sample data. In other words, it allows the extrapolation of the results of analysing a sample to the population that represents such sample (Moore 1996). 
Inferential tests are usually conducted to reject the null hypothesis $\left(\mathrm{H}_{0}\right)$ with respect to the alternative hypothesis $\left(\mathrm{H}_{1}\right)$, which is expected to be the cause of the phenomenon under study. This is determined through the $p$-value, which represents the probability of wrongly rejecting the null hypothesis if it is true. If the $p$-value is below the significance level, which can be defined as the threshold $\alpha$ to reject the null hypothesis, the probability of error is lower than a fixed value of $\alpha \%$ (Vergura et al. 2009). A value of $\alpha$ equal to 0.05 was used in this paper.

Normality of data distribution determined whether parametric (known distribution) or non-parametric (unknown distribution) statistical testing was applied. The Shapiro-Wilk test was used to check such normality, since it has proved to be more powerful than other commonly used tests such as Kolmogorov-Smirnov or Lilliefors (Razali and Wah 2011).

\subsubsection{Descriptive statistics}

Descriptive statistics refers to the procedures used to organize and characterize data collected from a sample or a population. Unlike inferential statistics, this discipline can only describe the group under study, not allowing the generalization of results to any larger group (Moore 1996).

Among the types of measures of descriptive statistics, a linear regression analysis was used in this study to estimate the relationships among geotextile-related variables influencing the biofilm growth. Five different assumptions must be met to ensure the validity of a linear regression model (Tabachnick and Fidell 1989):

(1) Linearity: the dependent variable is the sum of a set of elements: the intercept, a linear combination of the independent variables and the residuals.

(2) Independence: the residuals are independent from each other, i.e. they constitute a random variable.

(3) Homoscedasticity: the variance of the residuals is constant for each value of the independent variable (or combination of values of the independent variables). 
(4) Normality: the residuals are normally distributed for each value of the independent variable (or combination of values of the independent variables).

(5) Collinearity: there is no linear relationship between the independent variables.

\section{Results and discussion}

Two separate statistical analyses were carried out using the IBM SPSS Statistics 22.0 software (IBM Corp. 2013). The purpose of this section is to validate the conclusions deduced from the experimental values, while highlighting the underlying physical phenomena that might be leading to them.

\subsection{Test 1. Biofilm growth and development on geotextiles}

After each of the three times of extraction (15, 30 and 52 days), the biofilm weight developed in each geotextile sample was measured, resulting in the average values shown in Table 2.

Table 2. Biofilm weights measured on geotextiles

By mere observation, clear differences between the magnitudes of the mass of biofilm generated on heat-sealed and needle-punched geotextiles can be seen. One way to validate these differences is to use a statistical test for comparing the means of both groups. Using the Shapiro-Wilk test, it was found that the weights of biofilm at all three time periods were normally distributed for both groups ( $p$-values greater than 0.05 in all cases). Thus, the Student's t-test was used to compare the biofilm mass developed on heat-sealed and needle-punched geotextiles, revealing that the differences between them were statistically significant. Nevertheless, by examining the physical features of the geotextiles (see Table 1), the variable "Thickness" appeared to be an influencing factor. Indeed, if the ten geotextiles are grouped according to the median of "Thickness", what happens is that those manufactured by heat-sealing are below such value and those by needle-punching above it. For this reason, the idea of analysing the results separately on according to the manufacturing process was 
rejected, since such disassociation is actually explained by the thickness of the fabrics, which is a continuous variable.

The data were also evaluated using the three time horizons ( $\mathrm{K}$ dependent variables) in which the biofilm mass was measured. Mauchly's W value (0.492) confirms the sphericity of the variance matrix and hence the relevance of performing a repeated measures ANOVA. The pairwise comparisons obtained through Scheffé's method suggested that the biofilm weight significantly varied at 52 days ( $p$-values $=0.000)$, but not between 15 and 30 days ( $p$-value $=1.000$ ). Thus, subsequent calculations were performed for the data at 52 days, where there was a significant jump in the mass of biofilm and thus a better reflection of its development.

Once the results had been contextualized in general terms, biofilm growth was modelled according to geotextiles characteristics, for which a multiple linear regression analysis was conducted. "Material" was quantified as either 0 (Not PP) or 1 (PP) with the remaining independent variables ("Mass", "Pore size" and "Thickness") directly introduced through their numeric values. First, Cook's distances were studied to detect influential points. A value of $D=1$ is typically assumed as the threshold beyond which the influence of a point may be relevant (Cook and Weisberg 1982). Other authors decrease it to $D=4 / N, N$ being the number of observations (Bollen and Jackman 1985). For the geotextile samples studied here, Geodren Pes ARX 120 and Terratest TMA 125 slightly exceeded the more restrictive limit (0.433 and 0.465). Notice that these were the only woven and heat-sealed on one side types, respectively. These aspects were not taken into account initially, relying instead on the ability of the statistical analysis to highlight them. However, as the amount by which these values exceeded the threshold was practically negligible, none of these geotextiles were removed from the analysis, since the loss of representativeness of the sample could be more harmful than the inclusion of this pair of observations.

Among the assumptions a linear regression model must meet, collinearity was undertaken first, as it can lead to a situation wherein the outcomes of several of the 
remaining assumptions could be altered. Table 3 depicts the collinearity diagnostics of this model.

Table 3. Collinearity diagnostics between the geotextiles characteristics

Condition indices greater than 10 can be considered a symptom of collinearity (Belsley et al. 1980). Under this premise, up to three independent variables were affected by this phenomenon, with "Thickness" as the only explanatory variable to the model, as it did not present the highest variance proportion in any of the dimensions. Stated differently, the remaining variables ("Material", "Mass" and "Pore size") did not add further information to that provided by "Thickness" when explaining the developed biofilm mass. Regarding the material, the density of polyester $\left(1.38 \mathrm{~g} / \mathrm{cm}^{3}\right)$ was much higher than that of polypropylene $\left(0.91 \mathrm{~g} / \mathrm{cm}^{3}\right)$, which probably hindered the development of thin geotextiles (the two polyester samples were thickest). Unlike mass per unit area, which is also directly proportional to thickness, the pore size showed a negative Pearson's correlation with biomass (which is logical, since larger pore size involves less retention and therefore greater difficulty for biofilm accumulation). However, such interaction was not significant in statistical terms ( $p$-value $=0.226$ ), which supported the removal of this variable from the regression model. Simple regression analysis of Thickness ${ }^{*}$ Biomass returned the results shown in Table 4 and Figure 3.

Table 4. Summary of the regression model Thickness*Biomass

Figure 3. Analysis of residuals of the regression model Thickness*Biomass

The R square coefficient indicated that $92.4 \%$ of the variation of "Biofilm" is explained by "Thickness". This value was not affected by the size of the sample, as the adjusted $\mathrm{R}$ square coefficient only decreased by $1 \%$. As mentioned in 2.3.2, a linear regression model must meet four other assumptions in addition to collinearity: independence, linearity, normality and homoscedasticity. The first one was accepted in this case, as the Durbin-Watson statistic was between 1.5 and 2.5 (Durbin and Watson 1950; Durbin and Watson 1951). The p-value of F- in the ANOVA confirmed the hypothesis that the 
slope of the regression line was zero and therefore the linear relationship between the two variables. In turn, Figure $3 a$ ) and Figure $3 b$ ) graphically show how the standardized residuals were close to a normal distribution. Furthermore, homoscedasticity of residuals can also be assumed since there were no outliers among their variances (Figure 5c) and Figure 5d)).

\subsection{Test 2. Geotextiles resistance to microbiological degradation}

After a four-month period, as stated in 2.2.5, breakage of the samples was performed according to standard UNE-EN ISO 13934-1:1999 (AENOR 1999). Figure 4 and Figure 5 summarize the results of tensile strength (expressed by the values of breaking load in $\mathrm{N}$ ) and elongation $(\mathrm{mm})$ for each combination of treatment and storage, for both exposed (ES) and control samples (CS).

Figure 4. Breaking load $(\mathrm{N})$ of exposed and control samples

Figure 5. Elongation (mm) of exposed and control samples

The characteristics of both types of geotextile, their mass, thickness and pore size, had considerable influence on their mechanical properties. Indeed, Polyfelt TS-30 samples reached higher values in both tensile strength and elongation than Inbitex, as a result of their thicker, heavier and tighter structure. The values yielded by the two geotextiles were normal and homoscedastic for tensile strength, as reflected in the Shapiro-Wilk and Levene's tests, but not for elongation. A p-value of 0.000 was obtained for both characteristics by applying the Student's t- and the Mann-Whitney $U$ tests, which corroborates the previously discussed divergence. Thus, the remaining conditions were examined separately for each type of geotextile. Obviously, the analyses of the exposed and control samples were independent.

With respect to the exposed geotextiles, their behaviour was examined for each of the three treatment and burial conditions. Polyfelt TS-30 was analysed through a one-way ANOVA, since its value distributions fulfilled the assumptions of normality and homoscedasticity, whereas Inbitex's data required a non-parametric test such as 
Kruskal-Wallis. The results allowed rejection of the alternative hypothesis in both cases ( $p$-values $>0.05$ ), which meant that the three treatments produced no mechanical distortions in the exposed samples, regardless of their nature. This polyvalence suggested constant behaviour of geotextiles in different environments, where microbiological activity was capable of inhibiting more or less diffuse concentrations of hydrocarbons, such as those present in the three treatments.

The control samples were studied according to their treatment and storage conditions, divided into four different data groups: oil/oil-free and fridge/outside, for both Polyfelt TS-30 and Inbitex. As all these subsamples met the Shapiro-Wilk test of normality, their means were compared through the Student's t-test. The storage conditions significantly affected the tensile strength and elongation of Polyfelt TS-30, but not those of Inbitex, which may be related to their manufacture, since the latter is bonded by heat-sealing and the former by needle-punching. A thermal treatment such as heatsealing produces a hermetic state that might be responsible for the resistance of Inbitex to temperature and humidity variations, which cannot be assured by a needle-punched geotextile. On the other hand, the opposite occurred with respect to the type of treatment. Oil decreased the mechanical features of Inbitex, but had no statistically conclusive impact on Polyfelt TS-30. Inbitex is partially made of polyester, an oleophilic material, and has larger pore size, which may result in greater ease for the oil to adhere to the fibres of the geotextile. This, in turn, lubricates the fibres, which can reduce their mechanical properties.

Considering the previous analyses, the original data set was reduced according to the groups showing statistically significant differences. By applying Eqs. (1) and (2), strength and elongation losses were calculated by comparing the values between exposed and control samples, as shown in Table 5.

Table 5. Percentage variations in strength and elongation between exposed and control samples

In general, the exposure of a geotextile to a microbiological environment does not adversely affect its mechanical behaviour. The only case wherein one property was 
slightly reduced was the comparison between exposed and oil-free samples of Inbitex. This geotextile had a larger pore size and was not very tightly woven, which might favour its degradation by microorganisms as they penetrated into its structure. However, when looking at the other control state, the microorganisms' capacity in removing the effect of oil became clear. Polyfelt TS-30 proved to be $100 \%$ resistant to the microorganisms' impact due to its tightness, which favoured the retention process and allowed enough time for microorganisms to act. Moreover, this small retained extra mass might have reinforced some common points of failure of the sample, thus causing a slight increase in its strength and elongation values. Furthermore, despite the sensitivity of this geotextile to non-confinement conditions, its mechanical properties under exposure remained higher than those stored in a fridge.

\section{Conclusions}

Geotextiles are one of the most important components in the design of pervious pavement, since they allow both the separation of different layers and the retention of pollutants passing through it, such as hydrocarbons. Furthermore, most of the growth of biofilm responsible for degrading such pollutants is concentrated in the geotextile layer. This is due to its retention capacity, which provides microorganisms enough time to consume hydrocarbons. For these reasons, choosing a geotextile can make a difference in achieving quality standards of water purification.

The results of carrying out the test of biofilm development and growth suggested thickness was important in supporting the generation of biomass, although there might be other factors influencing this condition to some extent. This strong dependency is logical, since thicker geotextiles involve larger volumes of fabric for microorganisms to grow on. In this sense, as a result of their respective manufacturing processes, needlepunched fabrics are usually thicker than heat-sealed ones, which makes them more suited to support microbiological growth. However, the selection of a geotextile to act in a pervious pavement structure does not only depend on its capacity of removing pollutants, but also on its infiltration efficiency. Therefore, both aspects must be 
considered when choosing a specific geotextile, in order to achieve a balanced performance.

Moreover, regarding the test designed to check their resistance against microorganisms, the outcomes show an almost complete maintenance of properties by the two geotextile types subject to the experiment. Furthermore, they both proved to respond similarly under the three proposed burial conditions, regardless of how the oil was added. Nevertheless, the needle-punched fabric presents a more regular behaviour with respect to the control samples, by virtue of its tighter structure. The exposure of geotextiles, either needle-punched or heat-sealed, to the action of a microbial community not only does not significantly diminish their mechanical characteristics in comparison with a controlled environment, but even increases them if the contrast is performed in relation to situations without inoculated biological activity wherein the effect of oil or atmosphere is uncontrolled.

\section{Acknowledgments}

This study was financed by the Spanish Ministry of Economy and Competitiveness through the research projects FIDICA (Ref. REN2003-05278/TECNO) and RHIVU (Ref. BIA2012-32463) with funds from the State General Budget (PGE) and the European Regional Development Fund (FEDER). The authors also wish to express their gratitude to the companies and organizations that provided the products and equipment necessary for carrying out the tests: Horiba Spain, Fibertex Geotextiles, TMA Terratest Medio Ambiente, TenCate, Naue Fasertechnik, Geotextiles Danosa and Universidad de Cantabria, through its research groups of Environmental Engineering (GIA) and Ecology and its Laboratory of Geosynthetics (LAGUC).

\section{References}

AENOR. (1999). "Textiles. Tensile properties of fabrics. Part 1: determination of maximum force and elongation at maximum force using the strip method." UNE-EN ISO 13934-1:1999. 
AENOR. (2001a). "Geotextiles and geotextile-related products - Method for determining the microbiological resistance by a soil burial test." UNE-EN 12225:2001.

AENOR. (2001b). "Geotextiles and geotextile-related products. General tests for evaluation following durability testing." UNE-EN 12226:2001.

AENOR. (2001c). "Textiles. Determination of the resistance of cellulose-containing textiles to micro-organisms. Soil burial test. Part I: Assessment of rot-retardant finishing." UNE-EN ISO 11721-1:2001.

Aleksieva, Z., Ivanova, D., Godjevargova, T., and Atanasov, B. (2002). "Degradation of some phenol derivatives by Trichosporon cutaneum R57." Process Biochemistry, 37(11), 1215-1219.

Belsley, D. A., Kuh, E., and Welsch, R. E. (1980). Regression Diagnostics: Identifying Influential Data and Sources of Collinearity. Wiley, Michigan (U.S.A.).

Bollen, K. A., and Jackman, R. W. (1985). Regression diagnostics an expository treatment of outliers and influential cases. Sage Publications, Michigan (U.S.A.).

Bond, P. C., Pratt, C. J., and Newman, A. P. (1999). "A review of stormwater quantity and quality performance of permeable pavements in the UK." 8th International Conference on Urban Storm Drainage, Sidney (Australia), 248-255.

Castro-Fresno, D., Andrés-Valeri, V. C., Sañudo-Fontaneda, L. A., and RodriguezHernandez, J. (2013). "Sustainable Drainage Practices in Spain, Specially Focused on Pervious Pavements." Water, 5(1), 67-93.

Cook, R. D., and Weisberg, S. (1982). Residuals and influence in regression. Chapman and Hall, New York (U.S.A.).

Coupe, S. J., Smith, H. G., Newman, A. P., and Puehmeier, T. (2003). "Biodegradation and microbial diversity within permeable pavements." European Journal of Protistology, 39(4), 495-498.

Durbin, J., and Watson, G. S. (1951). "Testing for serial correlation in least squares regression. II." Biometrika, 159-177.

Durbin, J., and Watson, G. S. (1950). "Testing for serial correlation in least squares regression. I." Biometrika, 37(3-4), 409-428.

I.C.T, S. L. (2003). Manual básico de Microbiología - CULTIMED. Panreac Química S.A, Madrid (Spain).

IBM Corp. (2013). IBM SPSS Statistics for Windows, Version 22.0. IBM Corp., Armonk, New York (U.S.A). 
Li, Q., Kang, C., and Zhang, C. (2005). "Waste water produced from an oilfield and continuous treatment with an oil-degrading bacterium." Process Biochemistry, 40(2), 873-877.

Moore, L. M. (1996). "The basic practice of statistics." Technometrics, 38(4), 404-405.

Newman, A. P., Pratt, C. J., Coupe, S. J., and Cresswell, N. (2002). "Oil biodegradation in permeable pavements by microbial communities." Water Science and Technology, 45(7), 51-56.

Nnadi, E. O., Coupe, S. J., Sañudo-Fontaneda, L. A., and Rodriguez-Hernandez, J. (2014). "An evaluation of enhanced geotextile layer in permeable pavement to improve stormwater infiltration and attenuation." International Journal of Pavement Engineering, 15(10), 925-932.

Pratt, C. J., Newman, A. P., and Bond, P. C. (1999). "Mineral oil big-degradation within a permeable pavement: Long term observations." Water Science and Technology, 39(2), 103-109.

Razali, N. M., and Wah, Y. B. (2011). "Power comparisons of Shapiro-Wilk, Kolmogorov-Smirnov, Lilliefors and Anderson-Darling tests." Journal of Statistical Modeling and Analytics, 2(1), 21-33.

Tabachnick, B. G., and Fidell, L. S. (1989). Using multivariate statistics. Harper Collins, New York (U.S.A.).

U.S. EPA. (1994). "Automobile Emissions: An Overview." Rep. No. 5, U.S. Environmental Protection Agency Office of Mobile Sources, Ann Arbor, Michigan (U.S.A.).

Vergura, S., Acciani, G., Amoruso, V., Patrono, G. E., and Vacca, F. (2009). "Descriptive and inferential statistics for supervising and monitoring the operation of PV plants." IEEE Transactions on Industrial Electronics, 56(11), 4456-4464. 
Figures

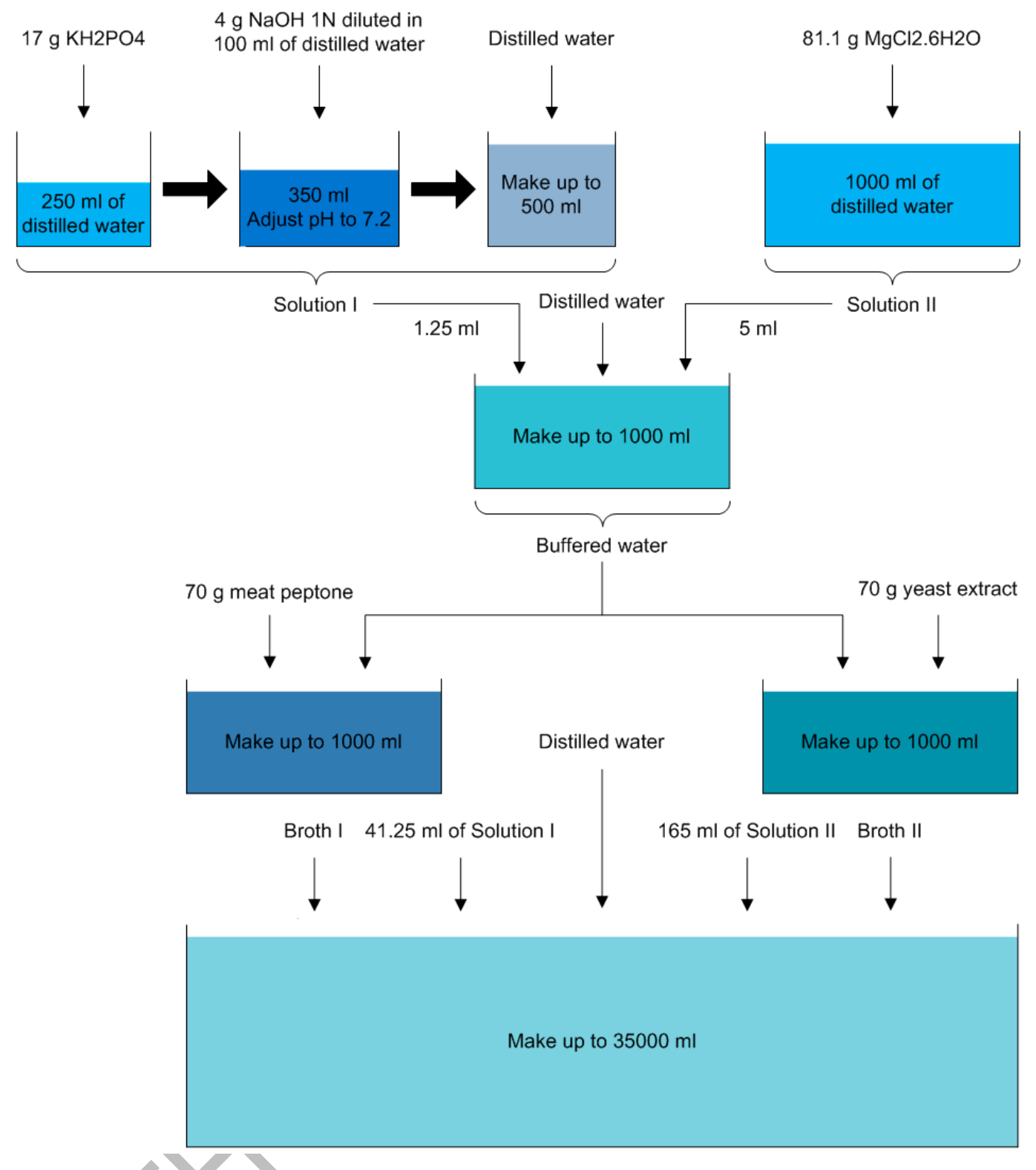

Figure 1. Preparation of the liquid medium in which the biofilm was grown on the tested geotextiles 

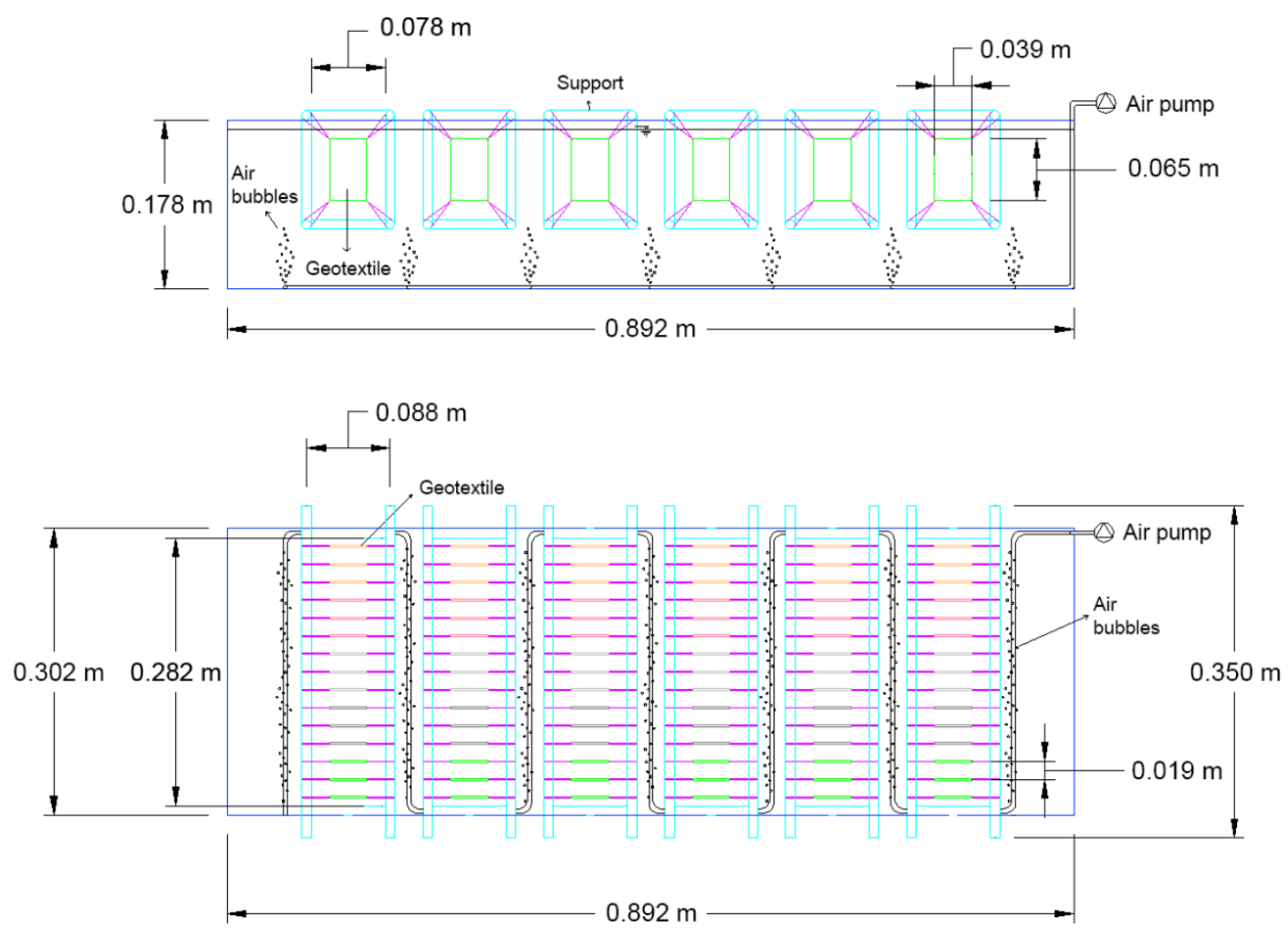

Figure 2. Front and plan view of the experimental set up 


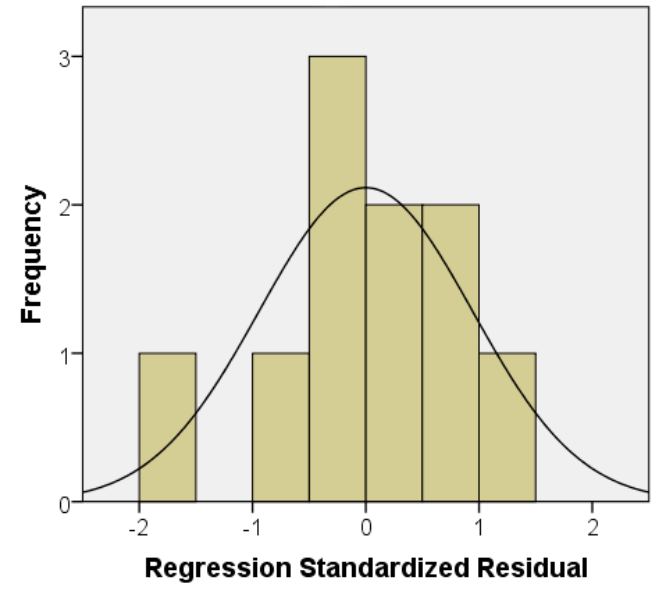

a) Histogram

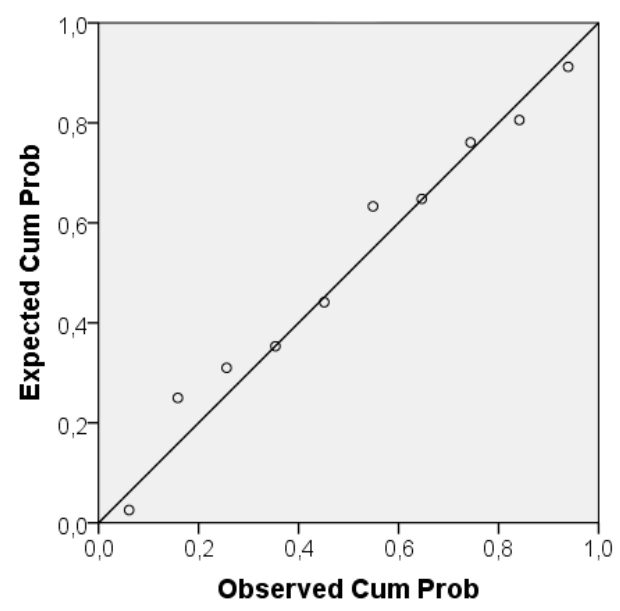

b) Normal P-P Plot

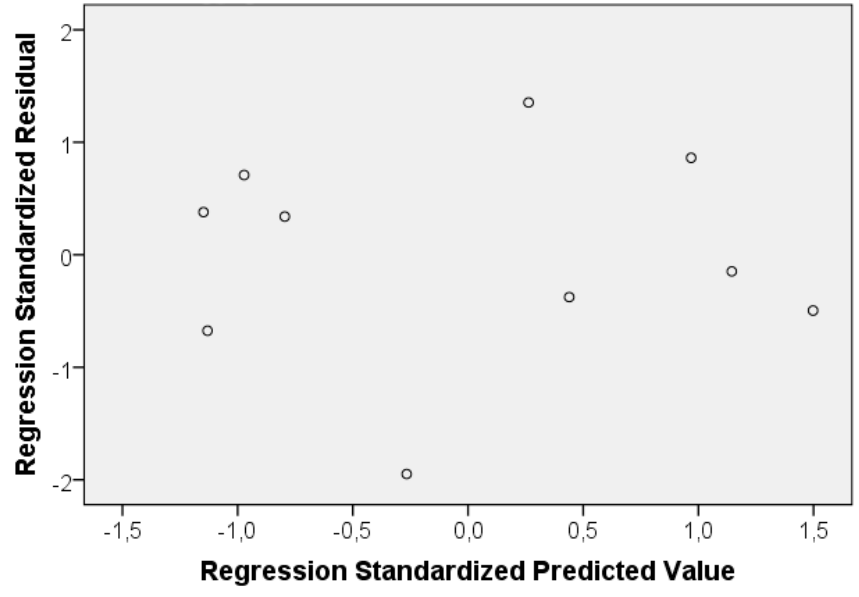

c) Scatterplot

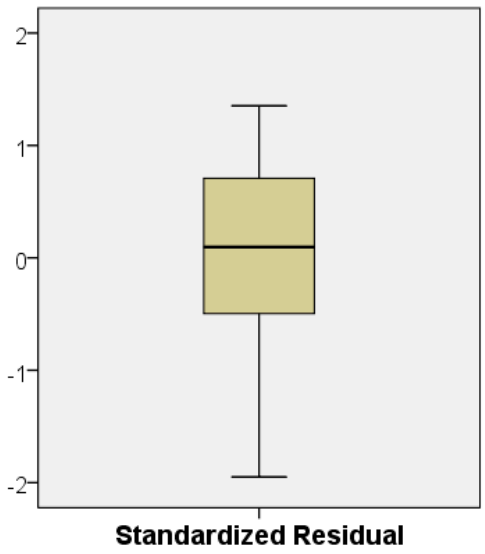

d) Outliers

Figure 3. Analysis of residuals of the regression model Thickness*Biomass 

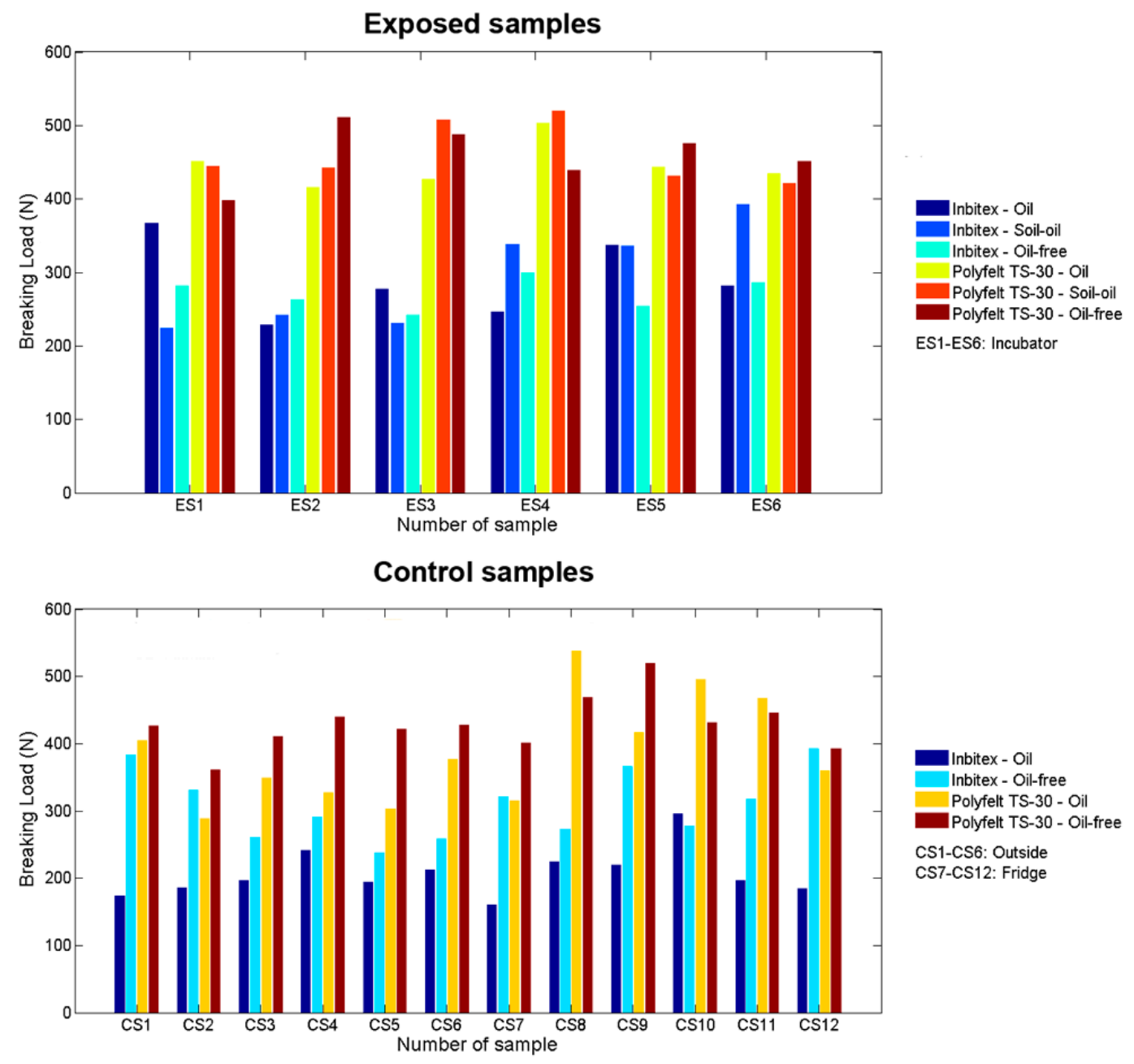

Figure 4. Breaking load $(\mathrm{N})$ of exposed and control samples 

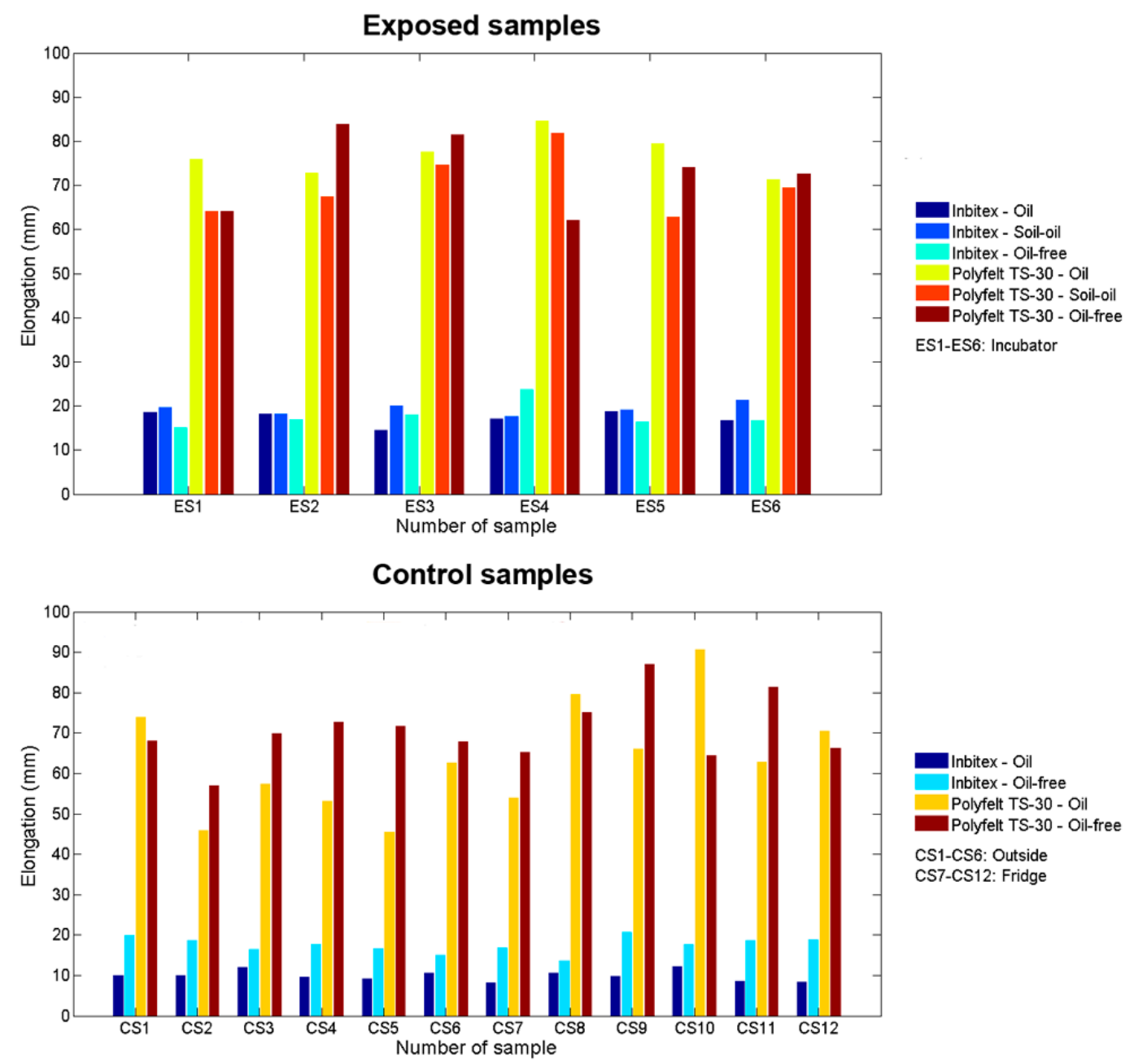

Figure 5. Elongation (mm) of exposed and control samples 


\section{Tables}

\begin{tabular}{lcccc}
\hline Geotextile & $\begin{array}{c}\text { Mass per unit } \\
\text { area }\left(\mathbf{g} / \mathbf{m}^{\mathbf{2}}\right)\end{array}$ & Material & Thickness (mm) & Pore size ( $\boldsymbol{\mu m})$ \\
\hline Geodren Pes ARX 120 & 120 & W / HS / PP & 0.61 & 160 \\
Polyfelt TS 30 & 155 & NW / NP / PP & 1.50 & 110 \\
Danofelt PP 125 & 125 & NW / NP / PP (70\%) & 0.70 & 150 \\
Danofelt PY 150 & 150 & NW / NP / PY & 1.90 & 100 \\
Danofelt PY 200 & 200 & NW / NP / PY & 2.10 & 90 \\
Terratest TMA 125 & 125 & NW / HS (1 side) / PP & 1.10 & 90 \\
Pavemat B (Amopave) & 140 & NW / NP / PP & 1.40 & 110 \\
Secutex 151 GRK 3 & 150 & NW / NP / PP & 1.80 & 130 \\
Fibertex G 100 & 100 & NW / HS / PP & 0.60 & 110 \\
Fibertex F2B & 140 & NW / HS / PP & 0.80 & 110 \\
\hline
\end{tabular}

W: Woven / NW: Nonwoven / HS: Heat-sealed / NP: Needle-punched / PP: Polypropylene / PY: Polyester

Table 1. Geotextiles characteristics 


\begin{tabular}{lccc}
\hline Geotextile & Biomass $_{\mathbf{1 5}} \mathbf{( g )}$ & Biomass $_{\mathbf{3 0}} \mathbf{( g )}$ & Biomass $_{\mathbf{5 2}} \mathbf{( g )}$ \\
\hline Geodren Pes ARX 120 & 0.0222 & 0.0298 & 0.0652 \\
Polyfelt TS 30 & 0.1022 & 0.0819 & 0.1490 \\
Danofelt PP 125 & 0.0445 & 0.0414 & 0.0944 \\
Danofelt PY 150 & 0.1031 & 0.1137 & 0.1881 \\
Danofelt PY 200 & 0.1100 & 0.1246 & 0.2006 \\
Terratest TMA 125 & 0.0510 & 0.0482 & 0.0892 \\
Pavemat B (Amopave) & 0.0998 & 0.0978 & 0.1667 \\
Secutex 151 GRK 3 & 0.0939 & 0.1168 & 0.1947 \\
Fibertex G 100 & 0.0295 & 0.0331 & 0.0805 \\
Fibertex F2B & 0.0360 & 0.0387 & 0.0977 \\
\hline
\end{tabular}

Table 2. Biofilm weights measured on geotextiles 


\begin{tabular}{|c|c|c|c|c|c|c|c|}
\hline \multirow[b]{2}{*}{ Model } & \multirow[b]{2}{*}{ Dimension } & \multirow[b]{2}{*}{ Eigenvalue } & \multirow{2}{*}{$\begin{array}{l}\text { Condition } \\
\text { Index }\end{array}$} & \multicolumn{4}{|c|}{ Variance Proportions } \\
\hline & & & & Material & Mass & Thickness & $\begin{array}{l}\text { Pore } \\
\text { size }\end{array}$ \\
\hline \multirow[t]{5}{*}{1} & 1 & 4.607 & 1.000 & 0.00 & 0.00 & 0.00 & 0.00 \\
\hline & 2 & 0.336 & 3.704 & 0.14 & 0.00 & 0.04 & 0.00 \\
\hline & 3 & 0.041 & 10.541 & 0.68 & 0.00 & 0.19 & 0.27 \\
\hline & 4 & 0.012 & 19.928 & 0.02 & 0.20 & 0.62 & 0.64 \\
\hline & 5 & 0.005 & 31.682 & 0.15 & 0.79 & 0.15 & 0.08 \\
\hline
\end{tabular}

Table 3. Collinearity diagnostics between the geotextiles characteristics 


\begin{tabular}{|c|c|c|c|c|c|c|}
\hline \multicolumn{7}{|c|}{ Model Summary } \\
\hline Model & $\mathrm{R}$ & R Square & $\begin{array}{l}\text { Adjusted R } \\
\text { Square }\end{array}$ & \multicolumn{2}{|c|}{$\begin{array}{l}\text { Std. Error of the } \\
\text { Estimate }\end{array}$} & $\begin{array}{l}\text { Durbin- } \\
\text { Watson }\end{array}$ \\
\hline 1 & 0.961 & 0.924 & 0.914 & & 153657 & 2.342 \\
\hline \multicolumn{7}{|c|}{ ANOVA } \\
\hline Model & & $\begin{array}{c}\text { Sum of } \\
\text { Squares }\end{array}$ & $\mathrm{df}$ & Mean Square & $\mathrm{F}$ & Sig. \\
\hline \multirow[t]{3}{*}{1} & Regression & 0.023 & 1 & 0.023 & 97.083 & 0.000 \\
\hline & Residual & 0.002 & 8 & 0.000 & & \\
\hline & Total & 0.025 & 9 & & & \\
\hline
\end{tabular}

Table 4. Summary of the regression model Thickness*Biomass 


\begin{tabular}{llrr}
\hline Geotextile & Comparison & Residual strength (\%) & Residual elongation (\%) \\
\hline \multirow{2}{*}{ Inbitex } & Exposed vs Oil Control & 137.5270 & 183.0109 \\
& Exposed vs Oil-free Control & 92.1333 & 103.2748 \\
\multirow{2}{*}{ Polyfelt TS-30 } & Exposed vs Fridge Control & 104.1191 & 101.9776 \\
& Exposed vs Outside Control & 120.5036 & 118.0505 \\
\hline
\end{tabular}

Table 5. Percentage variations in strength and elongation between exposed and control samples 